\title{
Gut microbiota profiles of commercial laying hens infected with tumorigenic viruses
}

\author{
Xianhua Wan ${ }^{1 \dagger}$, Laipeng X ${ }^{1 \dagger}$, Xiangli Sun ${ }^{1}$, Hui Li ${ }^{2}$, Fengbin Yan ${ }^{1,3}$, Ruili Han ${ }^{1,3}$, Hong Li ${ }^{1,3}$, Zhuanjian Li $i^{1,3}$, \\ Yadong Tian ${ }^{1,3}$, Xiaojun Liu ${ }^{1,3}$, Xiangtao Kang ${ }^{1,3}$, Zhenya Wang ${ }^{4^{*}}$ and Yanbin Wang ${ }^{1,3^{*}}$
}

\begin{abstract}
Background: Studies have shown that some viral infections cause structural changes in the intestinal microflora, but little is known about the effects of tumorigenic viral infection on the intestinal microflora of chickens.

Results: A 29-week commercial layer flock positive for avian leukosis virus-J (ALV-J), Marek's disease virus (MDV) and avian reticuloendotheliosis virus (REV) was selected, and fresh fecal samples were collected and examined for the composition of the gut microflora by Illumina sequencing of the V3-V4 region of the $16 \mathrm{~S}$ rRNA gene. The operational taxonomic units (OTUs) of the fecal microbiota differentiated the chickens infected with only ALV-J and those coinfected with ALV-J and MDV or REV from infection-negative chickens. The enrichment and diversity of cloacal microflora in chickens infected with ALV-J alone were slightly different from those in the infection-negative chickens. However, the diversity of cloacal microflora was significantly increased in chickens coinfected with both ALV-J and MDV or REV.

Conclusions: The intestinal microbiota was more strongly disturbed in chickens after coinfection with ALV-J and MDV or REV than after infection with ALV-J alone, and there may be underlying mechanisms by which the capacity for the stabilization of the intestinal flora was impaired due to viral infection and tumorigenesis.
\end{abstract}

Keywords: ALV-J, MDV, REV, Fecal microflora, $16 \mathrm{~S}$ rRNA

\section{Background}

Avian leukosis virus-J (ALV-J), Marek's disease (MD) virus (MDV) and avian reticuloendotheliosis virus (REV) are tumorigenic viruses that cause immunosuppressive, oncogenic and runting syndrome in layer chickens [1-3]. There are currently no commercial vaccines or effective drugs for controlling ALV-J and REV infections, resulting in great economic losses in the laying hen industry [4].

\footnotetext{
* Correspondence: zhenyawang@zzu.edu.cn; ybwang2008@henau.edu.cn †Xianhua Wan and Laipeng Xu are co-first authors

${ }^{4}$ Key Laboratory of "Runliang" Antiviral Medicines Research and

Development, Institute of Drug Discovery \& Development, Zhengzhou University, Zhengzhou 450001, China

${ }^{1}$ College of Animal Science and Veterinary Medicine, Henan Agricultural University, Zhengzhou 450000, China

Full list of author information is available at the end of the article
}

ALV-J is an avian retrovirus that was first isolated from meat-type chickens and mainly induces myelocytomatosis, nephromas and immunosuppression [5]. The prevalence of ALV-J, one of the major diseases in commercial layer flocks, has caused this dangerous disease to become a serious threat to the poultry industry worldwide, especially to local breeds in China in recent years [6-11]. Myeloid leukosis, hemangiomas and leiomyosarcomas are the main clinical manifestations of ALV-J that are observed simultaneously in commercial layer flocks with ALV-J infection $[12,13] . R E V$ is an avian retrovirus that causes lymphocyte tumorigenesis, immunosuppression, growth retardation and runting syndrome in chickens and turkeys [4]. REV may be transmitted horizontally by direct contact between birds and indirectly by mosquitos and contaminated

C C The Author(s). 2020 Open Access This article is licensed under a Creative Commons Attribution 4.0 International License, which permits use, sharing, adaptation, distribution and reproduction in any medium or format, as long as you give appropriate credit to the original author(s) and the source, provide a link to the Creative Commons licence, and indicate if changes were made. The images or other third party material in this article are included in the article's Creative Commons licence, unless indicated otherwise in a credit line to the material. If material is not included in the article's Creative Commons licence and your intended use is not permitted by statutory regulation or exceeds the permitted use, you will need to obtain permission directly from the copyright holder. To view a copy of this licence, visit http://creativecommons.org/licenses/by/4.0/ The Creative Commons Public Domain Dedication waiver (http://creativecommons.org/publicdomain/zero/1.0/) applies to the data made available in this article, unless otherwise stated in a credit line to the data. 
vaccines against fowlpox [14-16], Gallid herpesvirus 2 $[17,18]$ and MD [19]. Serologic studies have confirmed that reticuloendotheliosis viral infection is common in commercial layer, broiler, and turkey flocks worldwide [20-22]. The results of a serological survey in China from 2005 to 2015 showed that the virus-positive rate increased from 7 to $15 \%$, indicating that the virus has become common in commercial chicken flocks. Coinfection with ALV-J and REV has more severe consequences for growth, immunosuppression and mortality than infection with ALV-J or REV alone [23-25]. MD is a lymphoproliferative disease of chickens caused by an avian herpes virus, MDV. Currently, although there are vaccines available to prevent this disease, there is a clinically frequent occurrence of MD that may be due to the common occurrence of ALV-J infection, which leads to immunosuppression against vaccines [26]. The virus targets lymphoid tissue, such as the bursa of Fabricius, liver, and spleen, where tumors eventually develop, resulting in high mortality.

The interplay between microbial communities and hosts and interactions between microbes are essential for gut homeostasis [27, 28]. A balanced commensal gut microbiota is important to ensuring the health of the host [29]. The relationship between intestinal bacteria and host health has received widespread attention [3032]. Gut microbes lower the $\mathrm{pH}$ through metabolites, participate in carbohydrate metabolism and lipid metabolism through metabolic enzymes, provide nutrients to the host by synthesizing amino acids and vitamins, and nourish intestinal epithelial cells to strengthen the gut barrier by producing short-chain fatty acids [33]. Gut microbial communities are influenced by diet, gender, age, and breed in a dynamic stable state of equilibrium [34-36]. The equilibrium is easily broken upon attack by various pathogenic factors, which may lead to disease and reduced production performance. Many studies now report intestinal microbial changes after infection with a wide range of pathogens, including bacteria, fungi and their toxins, parasites and viruses, which results in a decrease in the abundance and diversity of the intestinal flora [37-42].

Yitbarek et al. reported that influenza viral infection in chickens results in a shift in the gut microbiota and disrupts host-microbial homeostasis. The use of probioticand/or fecal microbiota transplantation (FMT)-based interventions can promote chicken recovery from H9N2 infection [29]. Li et al. reported that infectious bursal disease virus (IBDV) is able to replicate in gut-associated lymphoid tissues (bursa, cecal tonsils and cecum), inducing histological lesions, strong local immune cell changes and alteration of the gut microbiota composition, which led to increased susceptibility to pathogens invading the gut [42]. Perumbakkam et al. also reported that MDV infection is able to induce changes in the core gut microbiome of chickens after the early and late cytolytic phases of viral replication [43]. Related to avian leucosis viruses (ALV), the cecal microbiome has been shown to differ significantly following infection of specific pathogen-free (SPF) chickens with viruses from the subgroups J or K [38]. However, little is known about the additive effects on the intestinal microflora in adult chickens and on the resulting immune status that may arise from coinfection in the same animal with more than one avian tumorigenic virus, which likely have consequences on global health and susceptibility to other diseases.

Some studies have shown that dual infection and triple infection with ALV-J and other viruses such as MDV are common [44] and that these multiple viral infections result in enhanced pathogenicity [45] and a high level of tumor appearance. Multiple infections with tumorigenic viruses are largely responsible for tumorigenesis [10]. It must be noted that tumorigenesis caused by ALV-J infection in poultry is a chronic process and a true multifactorial disease that has proven very difficult to reproduce experimentally in SPF birds [46].

Taking all these observations into account, we decided in the present study to select a Chinese commercial poultry farm flock where ALV-J outbreaks have been identified and deaths have been recorded in chickens with different degrees of tumor development associated with infection with MDV or REV. Then, layer hens were selected from this flock as being infected with ALV-J alone or coinfected with ALV-J and MDV or REV, and the microbial composition of feces was analyzed using high-throughput sequencing technology. The aim is to explore the effects of different avian tumorigenic viral infections on the chicken intestinal microbiota. The expectation is to lay the foundations to further explore the mechanisms underlying the interaction between the intestinal microbiota and avian tumorigenic viruses and the consequences on the health status.

\section{Results}

\section{Analysis of intestinal flora composition}

The intestinal microbial communities from three groups of 18 samples were identified at different levels.

In fecal samples from the chickens of the $\mathrm{N}$ group, the preponderant bacteria at the phylum level were Firmicutes (99.36\%). The main bacterial phyla of the chicken fecal infected with ALV-J were Firmicutes (99.55\%) and Proteobacteria $(0.20 \%)$. For the chickens infected with ALV-J and MDV or REV, the main bacterial phyla were Firmicutes (91.79\%) and Bacteroidetes (5.38\%) (Fig. 1).

At the genus level, the main intestinal bacteria of the $\mathrm{N}$ group were Lactobacillus (84.52\%), Streptococcus (12.03\%) and Enterococcus (1.58\%). The main intestinal 


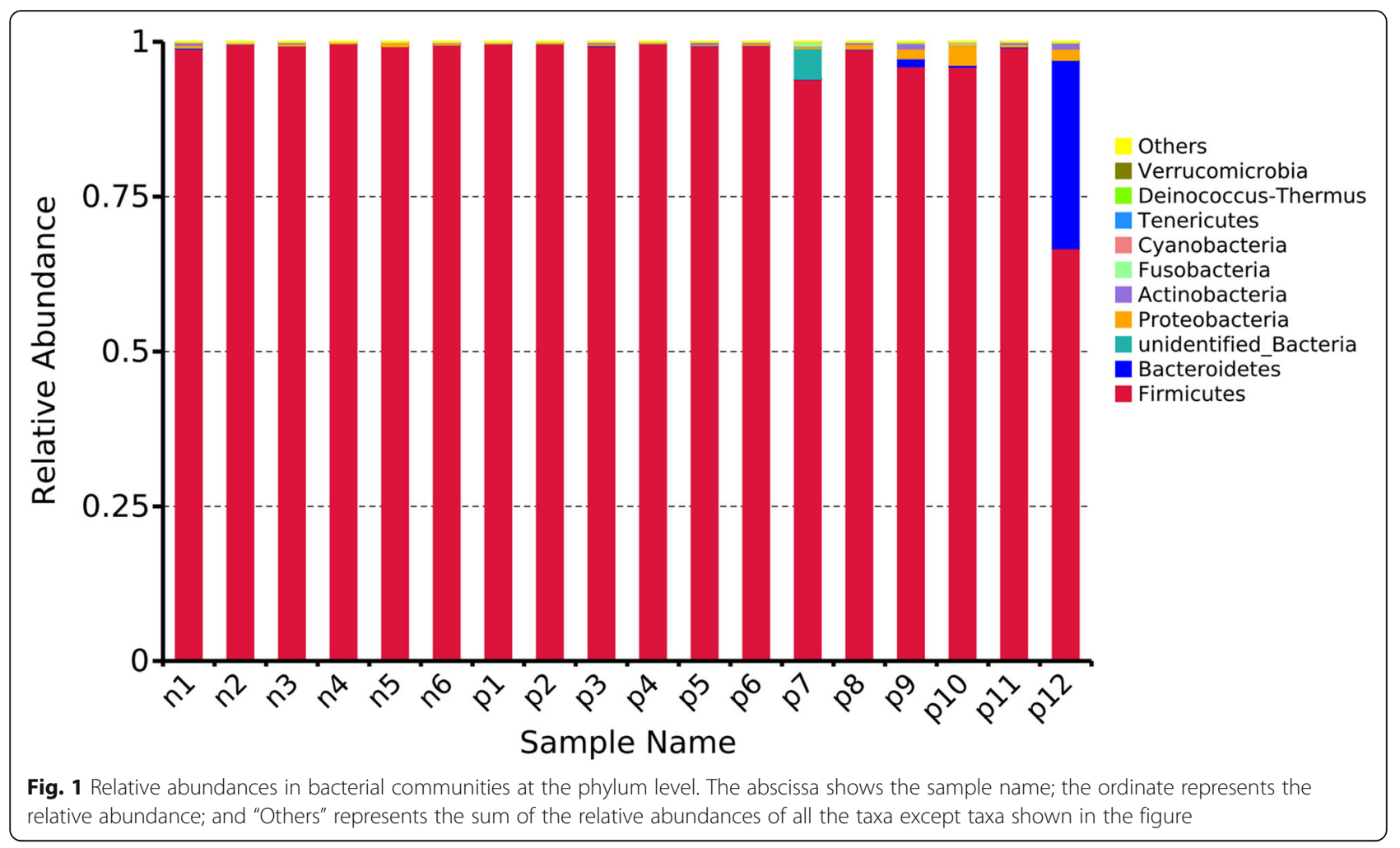

bacteria of the chicken P1 group were Lactobacillus (97.61\%), Streptococcus (1.18\%) and Enterococcus (0.15\%). Meanwhile, Lactobacillus (82.20\%), Bacteroides (3.43\%) and Enterococcus (2.94\%) were predominant in the chickens of the P2 group (Fig. 2).

Comparisons of intestinal bacterial communities at the phylum and genus levels among the three groups were performed. The results showed that there was no significant difference in the intestinal bacterial community between the three groups at the phylum and genus levels $(P>0.05)$.

Further analysis of the effective tags of groups N, P1, and P2 (230, 258 and 506 OTUs, respectively) showed that 155 OTUs were shared among all groups. Groups N and P1 shared 162 OTUs, groups N and P2 shared 211 OTUs, and groups P1 and P2 shared 239 OTUs. In addition, 12 OTUs were unique to group N, 12 OTUs were unique to group P1, and 211 OTUs were unique to group P2 (Fig. 3).

\section{Alpha-diversity index}

The microbial complexities in the gut of chickens were estimated on the basis of alpha-diversity indices (Chao1 indices and Shannon indices). Chaol was used to estimate species richness, while Shannon's index was used to indicate species diversity. The results showed that P2 samples had the largest alpha-diversity indices, followed by $\mathrm{P} 1$ and $\mathrm{N}$ (Table 1 ).

\section{Principal coordinate analysis}

A PCoA identifies the most important elements, and it extracts structure from multidimensional data through eigenvalue and eigenvector ordering. It can intuitively show whether the sample community structure of each group is different by the distance between the samples. As shown in Fig. 4, the distances between the 6 samples in group $\mathrm{N}$ were relatively small, indicating that the community difference was small; samples in groups P1 and P2 were relatively scattered, and the distances between samples were relatively large, indicating a large difference in communities in samples P1 and P2.

\section{Principal component analysis}

PCA revealed that infection with ALV-J and MDV or REV at the same time altered the bacterial communities in the gut of chickens when compared to uninfected chickens and chickens infected with only ALV-J (Fig. 5). Clear differences were observed in the bacterial communities between the N and P2 groups. However, no clear differences were observed in the community composition of bacteria in the gut samples between chickens infected with only ALV-J and uninfected chickens. Overall, the intestinal microbiota was more strongly disturbed in chickens after coinfection with ALV-J and MDV or REV than after infection with ALV-J alone. 


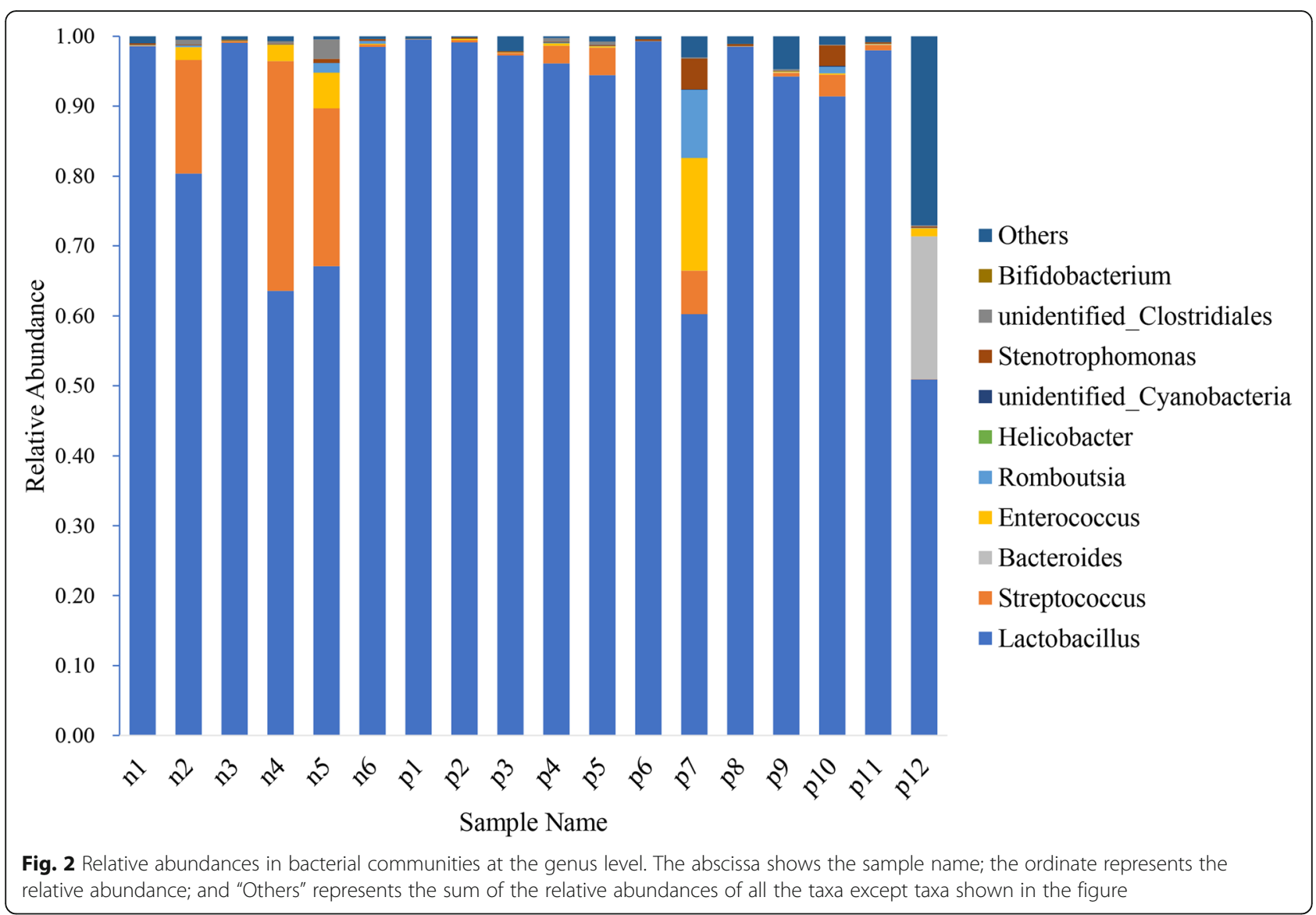

\section{Cluster heatmap of the relative abundances of species}

A heatmap of the 20 most abundant phyla was constructed for comparative analysis (Fig. 6). The composition of the intestinal microbiota showed obvious similarity based on ALV-J infection and healthy chickens, but for individuals, $\mathrm{n} 1, \mathrm{n} 6$ and $\mathrm{p} 5$ showed greater differences. However, in the samples infected with ALV-J and MDV or REV, there were abundant and obvious differences in multiple phyla between samples. The stable phylum in the first two groups also changed to varying degrees, and the phylum of the $\mathrm{p}_{12}$ sample was the most obviously changed. Sample p12 presented increases in the phyla Deinococcus-Thermus, Actinobacteria, Bacteroidetes, Gemmatimonadetes and Elusimicrobia, while the phylum Firmicutes decreased obviously.

\section{Column chart of the relative abundances of functional annotations}

To investigate the influence of tumorigenic viral infection on the functional performance of the microbiota, we performed Tax4Fun analysis of the three groups. As shown by the column chart of functional annotations based on the abundance of functional information in the annotation hierarchy (Fig. 7), we compared predicted microbial functions among three groups and detected that chemoheterotrophy and fermentation were most enriched. Although viral infections did not cause significant changes in these two main functions of the gut, other functions were affected to a certain extent. Chickens infected with ALV-J and MDV or REV had more intestinal microbial dysfunction than those infected with only ALV-J.

\section{Discussion}

Comparing gut microbiota composition in layer hens from a commercial flock shown to be infected by three different tumor-inducing avian viruses (ALV-J, MDV and REV), we established that the gut microbiota balance was disturbed differentially according to the infection by one (ALV-J, P1 group) or several (ALV-J and REV or MDV, P2 group) viruses compared to that of the control group (uninfected and apparently healthy, $\mathrm{N}$ group). The number of OTUs in the $\mathrm{P} 2$ group was much larger than those in the $\mathrm{N}$ and $\mathrm{P} 1$ groups. Moreover, the diversity of the flora was increased in the case of coinfection compared with infection with ALV-J alone. Our results corroborate the existing literature on intestinal microbiota changes following infection with avian tumor-inducing viruses. We further demonstrated that simultaneous infections by different viruses result in 


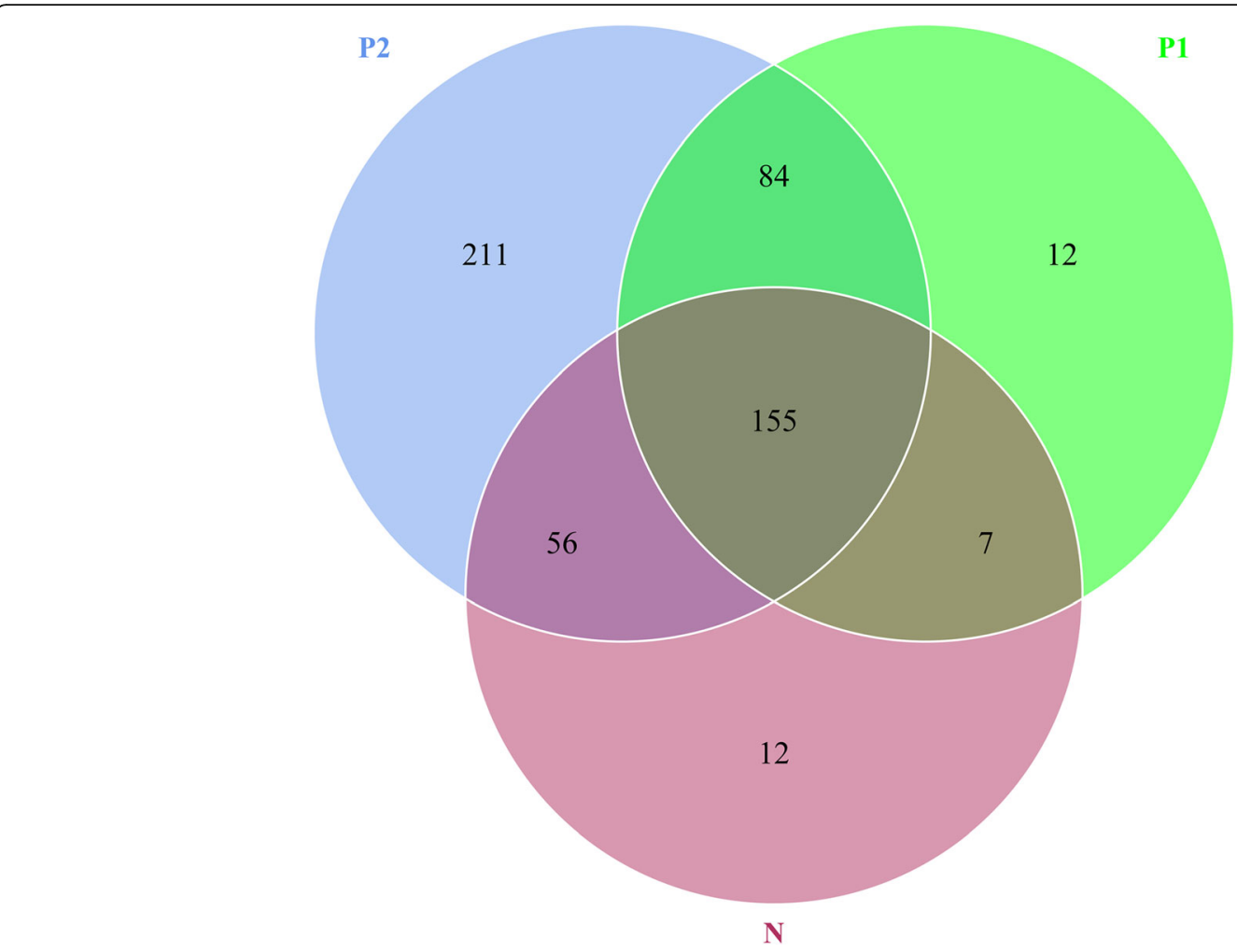

Fig. 3 Venn diagram of the intestinal flora structure. Each circle in the figure represents a sample, and the numbers in overlapping circles show the overlap between representative samples. The numbers of OTUs in nonoverlapping circles represent the numbers of unique OTUs in the samples

Table 1 Alpha-diversity indices from samples

\begin{tabular}{|c|c|c|c|c|}
\hline Sample name & Chaol & ACE & Simpson & Shannon \\
\hline$n_{1}$ & 174.25 & 185.867 & 0.65 & 2.314 \\
\hline $\mathrm{n}_{2}$ & 115.4 & 123.013 & 0.796 & 2.837 \\
\hline$n_{3}$ & 111.65 & 121.056 & 0.809 & 2.816 \\
\hline $\mathrm{n}_{4}$ & 103.053 & 110.75 & 0.78 & 2.79 \\
\hline $\mathrm{n}_{5}$ & 111.65 & 120.15 & 0.846 & 3.253 \\
\hline${ }^{n} 6$ & 112.5 & 116.492 & 0.586 & 2.105 \\
\hline$p_{1}$ & 121.067 & 118.74 & 0.693 & 2.281 \\
\hline$p_{2}$ & 100.5 & 106.726 & 0.648 & 2.012 \\
\hline$p_{3}$ & 181.812 & 195.909 & 0.767 & 2.746 \\
\hline $\mathrm{p}_{4}$ & 103.048 & 112.166 & 0.787 & 2.738 \\
\hline$p_{5}$ & 120.6 & 128.981 & 0.791 & 2.825 \\
\hline$P_{6}$ & 96.455 & 101.994 & 0.709 & 2.569 \\
\hline$p_{7}$ & 153.84 & 156.115 & 0.888 & 3.656 \\
\hline$p_{8}$ & 166.533 & 186.451 & 0.775 & 2.732 \\
\hline$p_{9}$ & 273.5 & 277.218 & 0.832 & 3.262 \\
\hline $\mathrm{p}_{10}$ & 190.333 & 188.509 & 0.733 & 2.763 \\
\hline $\mathrm{P}_{11}$ & 77.929 & 80.859 & 0.704 & 2.32 \\
\hline $\mathrm{p}_{12}$ & 359.111 & 360.692 & 0.936 & 5.317 \\
\hline
\end{tabular}




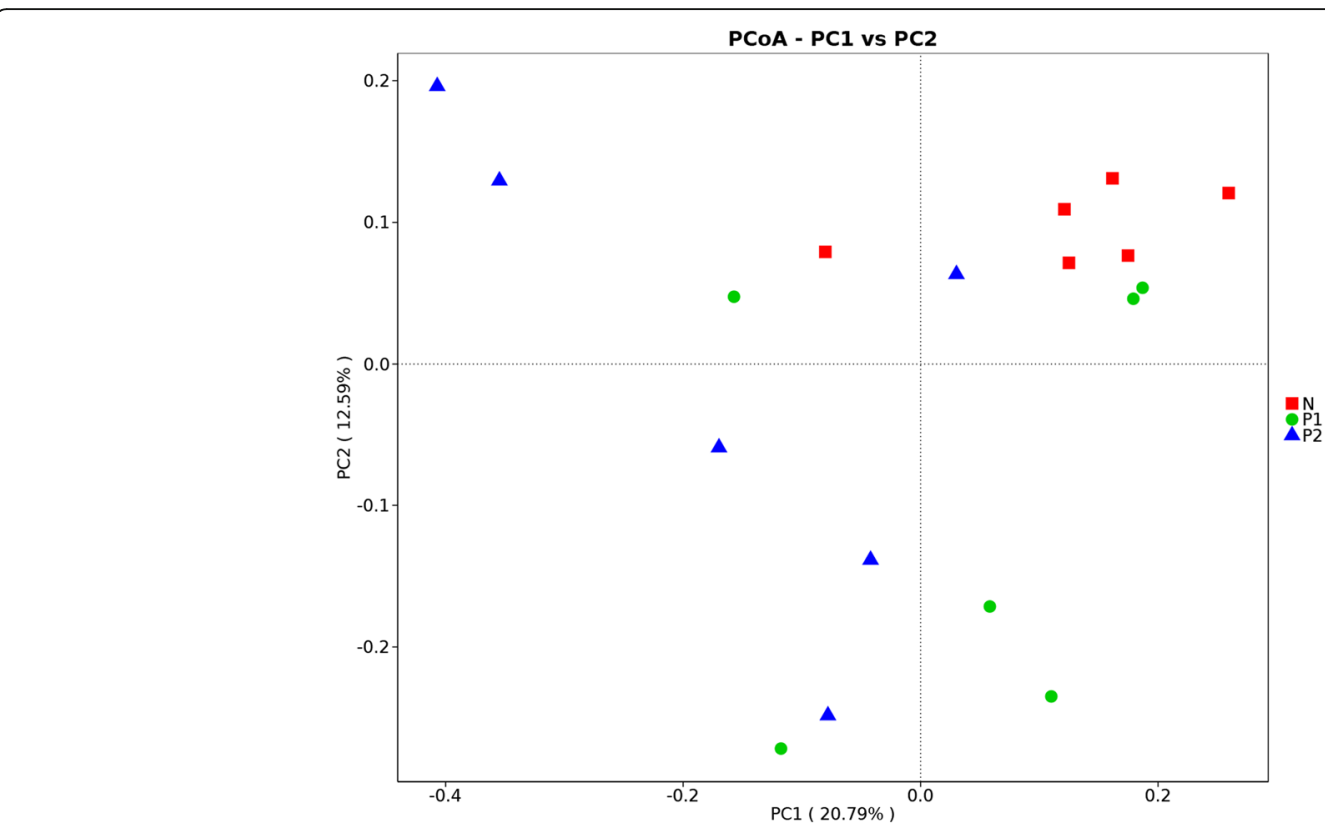

Fig. 4 Principal coordinate analysis. The abscissa and ordinate each represent a principal component, and the percentage represents the amount of variation between samples explained by the principal component; each point in the figure represents a sample, and the samples in the same group are shown in the same color

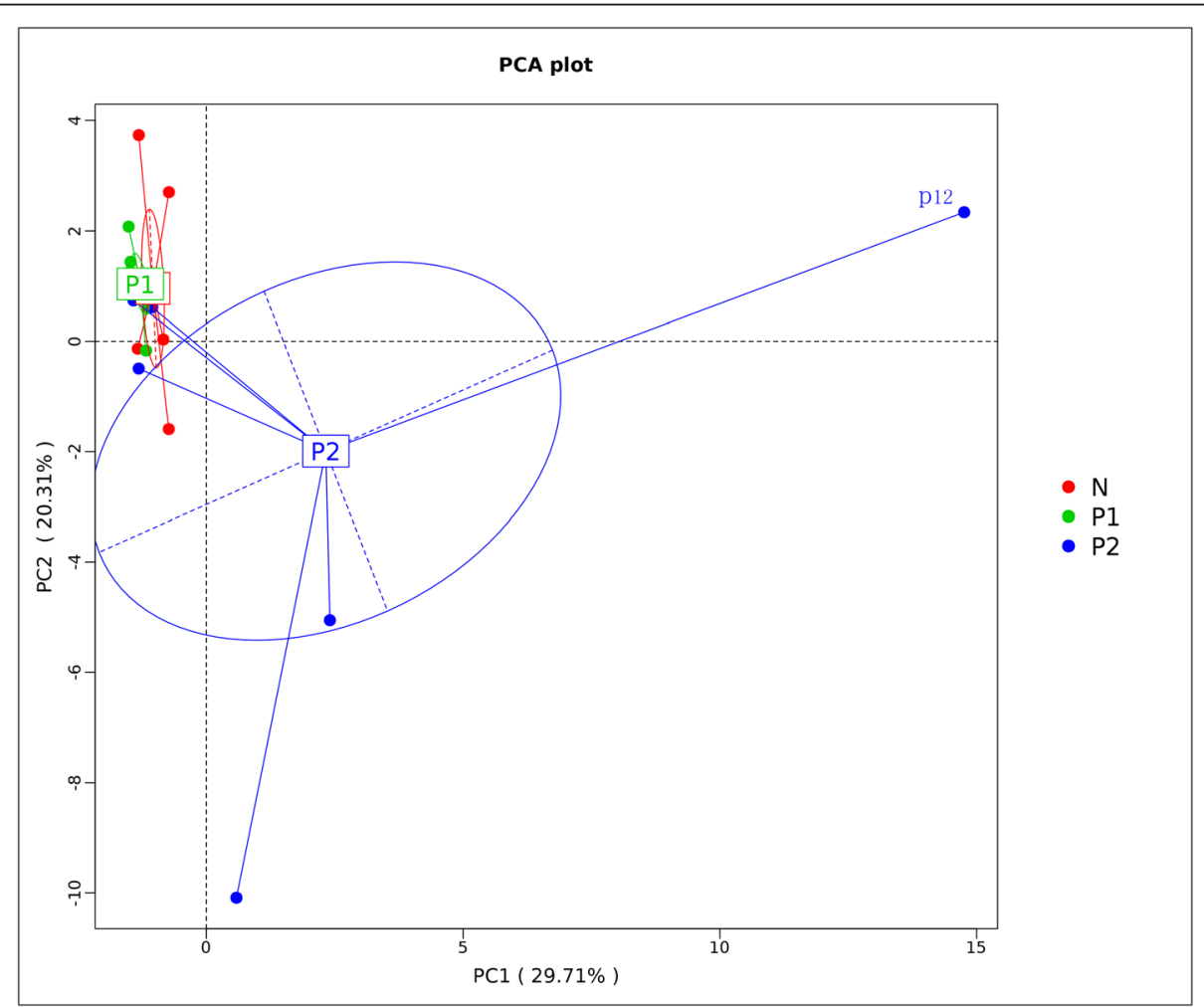

Fig. 5 Principal component analysis diagram. Samples with more similar flora structures are closer in distance; otherwise, the distance between samples is greater 


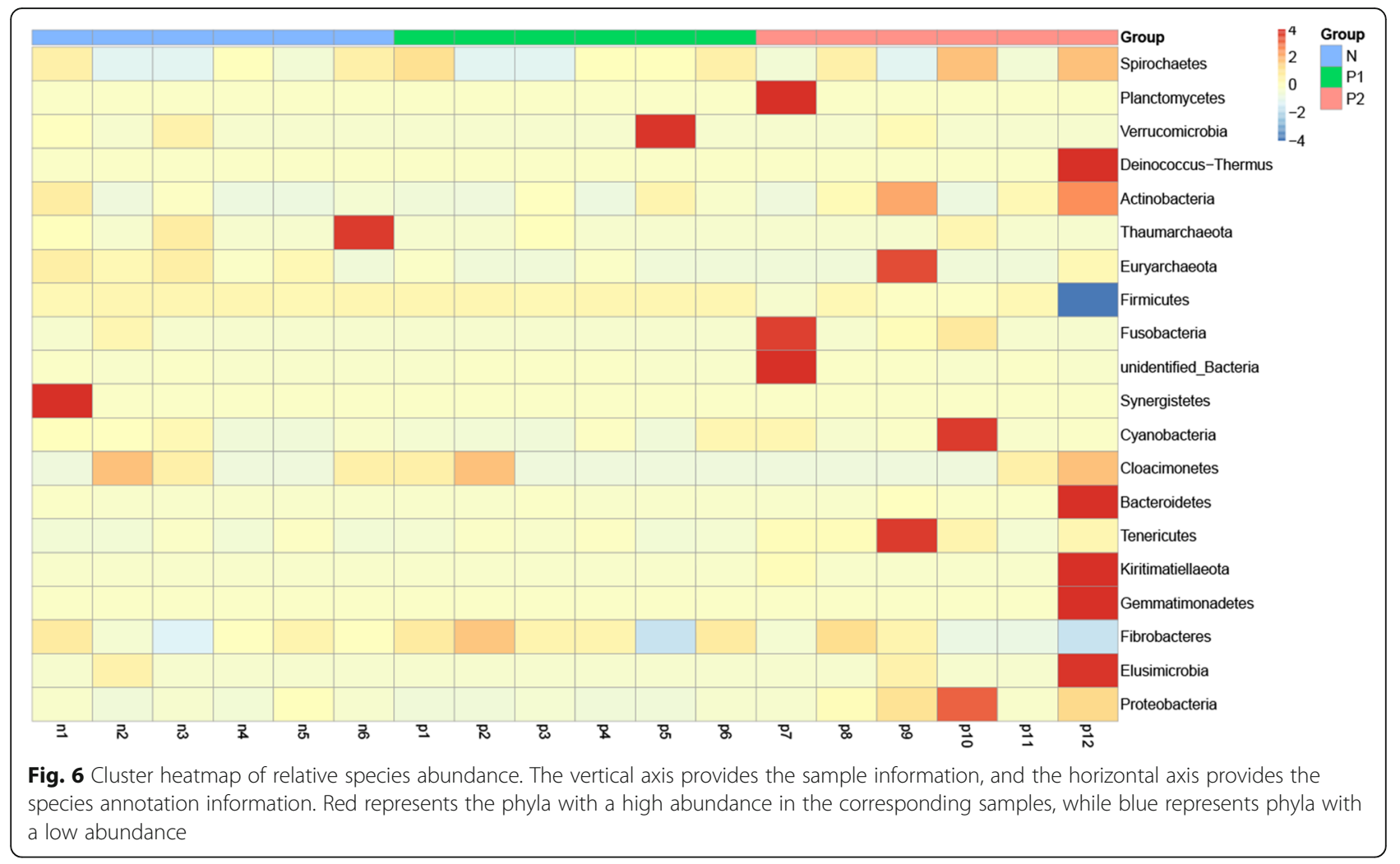

more severe disturbances of the gut microbiota composition [38, 43, 47]. The PCoA results showed a large difference in the fecal microbiota communities of tumorigenic virus-infected chickens, suggesting that the fecal microbiota compositions were changed by tumorigenic viral infection. The PCA displayed consistent results with the PCoA in classifying the fecal samples. In addition, the PCA results showed that the fecal microbiota compositions of dually infected individuals varied more than those of the ALV-J-infected individuals.

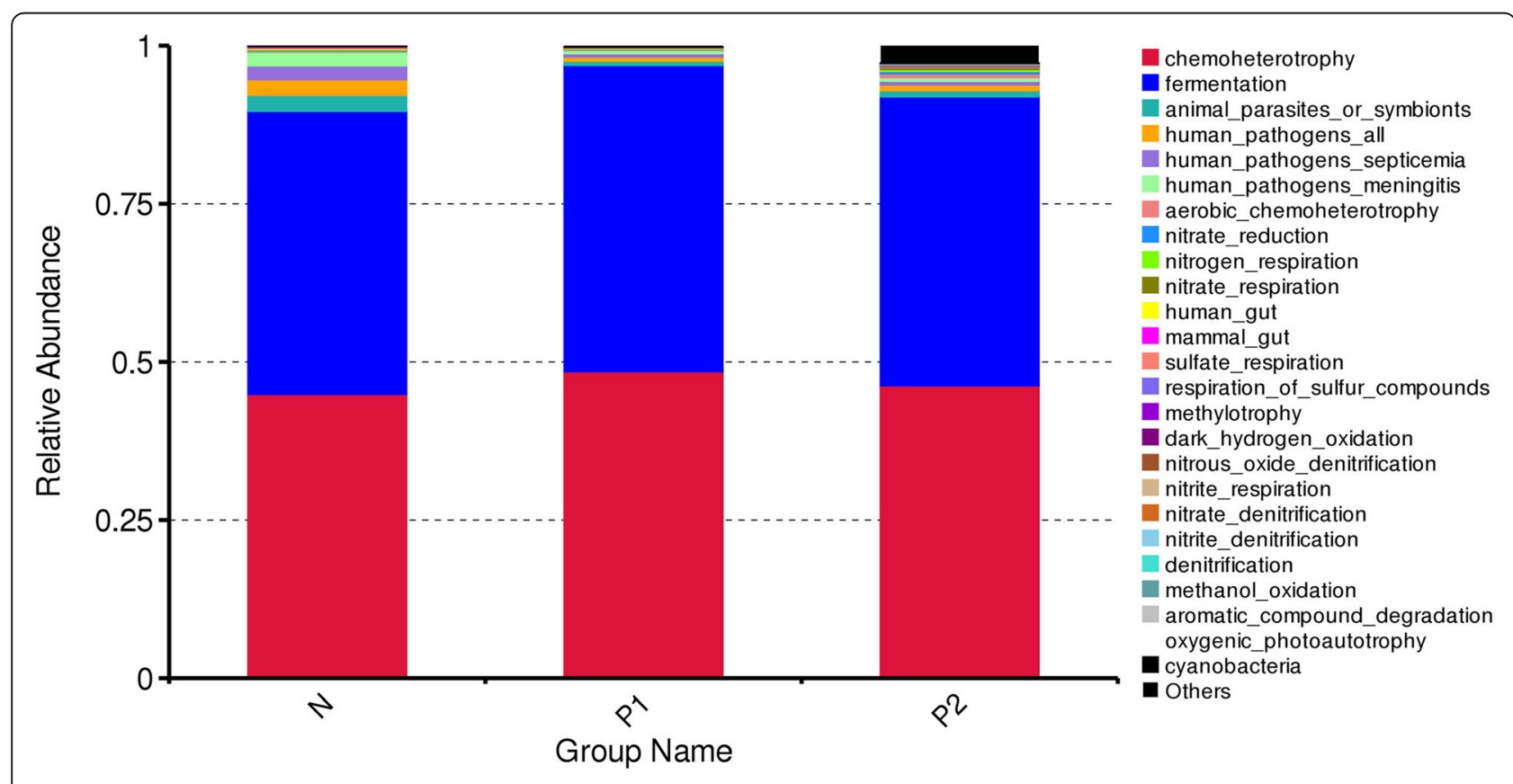

Fig. 7 Column chart of the relative abundances of the Tax4Fun functional annotations. Different colors represent different functional annotations 
This pattern of intestinal flora disorders associated with infection with ALV-J alone or coinfection with different avian tumor-inducing viruses might be supported by the appearance of physical/functional digestive disorders induced by these viruses and the alteration of the dialog between the host and the gut microbiota through immunosuppression [38, 43, 47]. The changes in the intestinal microbiota balance may favor infection with pathogens from the digestive tract and negatively affect chicken health. Intestinal epithelial cells infected by various pathogens easily lose their integrity, enabling microbes and metabolites to translocate and invade mucosal vessels and thus cause systemic infection and septicemia $[47,48]$. This means that gut intestinal changes after infection by ALV-J may favor the infection/replication of MDV and/or REV and tumor development, thus exacerbating the disease and its consequences.

Specific changes that may be observed in the gut microbiota composition after tumor-inducing infection have been observed in previous studies. The gut microflora from ALV-J predominantly includes opportunistic pathogens in Firmicutes, such as members of Staphylococcus and Weissella, and some genera of Bacillales. Moreover, [38] opportunistic pathogens such as Escherichia-Shigella and Enterococcus, members of Erysipelotrichaceae in Firmicutes and members of Helicobacteraceae in Bacteroidetes increase sharply in ALV-J-infected chickens. We hypothesized that the effect of viral infection on the intestinal microbial community mainly depends on the tissue tropism of the virus for target organs [49]. Studies on ALV-J-induced pathology have shown that the virus induces the formation of several types of tumors, such as hemangioma, leiomyosarcoma and myeloma (immune system). Thus, similar to REV and MDV, ALV-J most likely induced immunosuppression as the tumors progressed $[11,50]$. The clinical course of RE and MD neoplastic diseases targeting the immune system may be acute and chronic, as for ALVJ-induced disease. The viruses cause immunosuppression through depletion of lymphocytes for MDV and REV and tumorigenesis affecting $\mathrm{T}$ lymphocytes for MDV and B lymphocytes for REV in natural conditions [1]. Accordingly, this broad immunosuppression may strongly impact the gut immune system-microbiota balance. Moreover, solid lymphoid tumors may localize in different organs, including mostly lymphoid tissues (bursa, spleen) but also frequently the liver. In addition, lesions of the digestive tract, such as proventriculus lesions, enteritis and liver necrosis may be observed, especially for REV [49], which probably disturbs gastrointestinal functioning. Thus, it may also be hypothesized that this is another important cause of gut microbiota imbalance. In conclusion, we strongly suppose that simultaneous infection by more than one tumor-inducing virus in chickens has an additive effect on the immunosuppressed status and the alteration of gut function and integrity, leading to disruption of the dialog between the microbiota and the host, and to increased microbiota disturbances, as we observed [4, 24, 51].

In addition to an increase in the abundance of opportunistic bacteria, a large number of microorganisms that normally do not reside in the gut or reside at very low levels such as Cyanobacteria and Actinobacteria were found to increase in chickens with double infection by ALV-J and MDV or REV [52]. These results are consistent with those of a report [53] showing that the diversity of the cecal microflora increased in chickens infected with ALV-J. By contrast, when referring to other pathogens inducing serious damage to intestinal tissues and causing enteritis and diarrhea, such as Eimeria tenella, a sharp decrease of the diversity of the cecal microflora [53]. Thus, infection with several tumor-inducing viruses that are strongly deleterious to the immune system may differently impact the dialog between the host and the microbiota at the gut level, resulting in increasing bacterial diversity, although being able to alter somehow the gut integrity.

Chickens infected chronically with ALV-J exhibit most of the time normal feed and water intake, and no interruption of egg laying $[6,8]$. By contrast, increased alteration of normal gut function that may follow coinfection with several tumor-inducing viruses, as previously described, may result in alteration of feed intake and decreases in nutrients favorable to gut microbiota richness. There were differences in the functional annotation of the intestinal microorganisms between chickens infected with ALV-J alone and chickens with coinfections of ALV-J and MDV or REV. This difference is probably due to the weak intestinal microbiota stability and vulnerability to exogenous bacteria, leading to functional disorders. Therefore, mixed infections are more likely to cause substantial changes in the number and species of flora than are single ALV-J infections and lead to structural disorders in the chicken flora.

\section{Conclusion}

This study is the first to compare the effects of infection with ALV-J and coinfection with other tumor-inducing viruses, MDV and REV, on layer hens, as commonly observed in the field. We confirmed that infection with ALV-J can lead to gut microbiota structural changes. The main manifestations were a decrease in the phylum Firmicutes and an increase in the phyla Bacteroidetes and Proteobacteria and unidentified bacteria. We show that coinfection increased the diversity of unclassified bacteria and Cyanobacteria and Actinobacteria but reduced the richness of the dominant members of the flora usually present in noninfected chickens, despite 
none of the chickens displaying any symptoms. This let us suppose an alteration of the dialog between the host and the gut microbiota, presumably due to additive effects of these viruses through persistent immunosuppression and increased gut function alteration and lesions. Further studies are needed to understand how the improvement of the gut microbiota imbalance by appropriate diets and/or probiotics may be beneficial to resistance to avian chronic neoplastic diseases.

\section{Methods}

\section{Experimental design}

Eighteen 29-week-old commercial laying hens were selected according to their different infections, of which six healthy chickens formed the negative control group and were numbered n1-n6; six chickens infected with ALV-J formed group P1 and were numbered p1-p6; and six ALV-J- and MDV- or REV-infected chickens formed group P2 and were numbered p7-p12. Chickens infected with ALV-J and MDV- or REV were diagnosed by nucleic acid detection and serological examination at the College of Veterinary Medicine, Henan Agricultural University [54-56]. After being selected, each of the chickens was isolated in a cage under the same environmental conditions for 2 weeks and return to the farmer after the experiment.

All chickens were immunized against common diseases such as MD, infectious bursa disease, Newcastle disease and infectious bronchitis in accordance with established immunization procedures. The MDV vaccine was CVI988 liquid nitrogen vaccine, which was inoculated at the age of 1 day. The mortality rate of the chickens at 190 days of age was 5.2\% (512/9847) for the entire flock. The anatomy of randomly selected dead chickens revealed the main manifestations of hemangioma (26/ $32)$, liver tumor (9/32), spleen tumor (5/32), proventriculus tumor (3/32) and other tumors (5/32). Cases presented alone and in combination accounted for almost $100 \%$ of the deaths.

\section{DNA extraction}

All fecal samples were collected from the cloaca using sterile cotton swabs every $2 \mathrm{~h}$ from 6:00 a.m. to 18:00 p.m. and immediately placed into sterile conical tubes, frozen in liquid nitrogen, and then stored at $-80^{\circ} \mathrm{C}$ until use. Samples from each chicken were mixed before use. Microbial genomic DNA extraction was carried out with a ZR fecal DNA kit (Invitrogen, Carlsbad, CA, USA) according to the manufacturer's instructions. The quantity and quality of extracted DNA were measured using a NanoDrop ND-1000 spectrophotometer (Thermo Fisher Scientific, Waltham, MA, USA) and agarose gel electrophoresis, respectively.

\section{S rRNA amplification and MiSeq sequencing}

Polymerase chain reaction (PCR) amplification of the bacterial 16S rRNA gene V3-V4 region was performed using the forward primer 338F (5'-ACTCCTACGGGA GGCAGCA-3') and the reverse primer 806R (5'GGACTACHVGGGTWTCTAAT-3'). Sample-specific 7-bp barcodes were incorporated into the primers for multiplex sequencing. The PCR components included $5 \mu \mathrm{l}$ of Q5 reaction buffer (5x), $5 \mu \mathrm{l}$ of Q5 High-Fidelity GC buffer $(5 \times), 0.25 \mu \mathrm{l}$ of Q5 High-Fidelity DNA Polymerase $(5 \mathrm{U} / \mu \mathrm{l}), 2 \mu \mathrm{l}(2.5 \mathrm{mM})$ of dNTPs, $1 \mu \mathrm{l}(10 \mu \mathrm{M})$ of each forward and reverse primer, $2 \mu \mathrm{l}$ of DNA template, and $8.75 \mu \mathrm{l}$ of $\mathrm{dd}_{2} \mathrm{O}$. Thermal cycling consisted of initial denaturation at $98^{\circ} \mathrm{C}$ for $2 \mathrm{~min}$, followed by 25 cycles of denaturation at $98^{\circ} \mathrm{C}$ for $15 \mathrm{~s}$, annealing at $55^{\circ} \mathrm{C}$ for $30 \mathrm{~s}$, and extension at $72{ }^{\circ} \mathrm{C}$ for $30 \mathrm{~s}$, and a final extension of $5 \mathrm{~min}$ at $72^{\circ} \mathrm{C}$. PCR amplicons were purified with Agencourt AMPure Beads (Beckman Coulter, Indianapolis, IN, USA) and quantified using a PicoGreen dsDNA Assay Kit (Invitrogen, Carlsbad, CA, USA). After the individual quantification step, amplicons were pooled in equal amounts, and paired-end $2 \times 300 \mathrm{bp}$ sequencing was performed using the Illumina MiSeq platform (San Diego, CA, US) with a MiSeq Reagent Kit v3 (San Diego, CA, US) at Shanghai Personal Biotechnology Co. Ltd. (Shanghai, China).

\section{Sequence analysis and bioinformatic analysis}

The quantitative insights into microbial ecology (QIIME, v1.8.0) pipeline was employed to process the sequencing data, as previously described [57]. Low-quality sequences were filtered out through the following criteria [58, 59]: sequences with length of $<150 \mathrm{bp}$, average Phred scores of $<20$, ambiguous bases, and mononucleotide repeats of $>8 \mathrm{bp}$. Paired-end reads were assembled using FLASH (v 1.2.7, http://ccb.jhu.edu/software/FLASH/) [60]. The remaining high-quality sequences were clustered into operational taxonomic units (OTUs) at 97\% sequence identity by UCLUST (v0.2.0) of QIIME, and OTUs with abundance less than $0.001 \%$ of the total sequences were discarded [61]. The taxonomic information for the representative sequence in each OTU was obtained by matching the sequence database using BLAST of QIIME (v1.8.0).

Alpha-diversity indices (i.e., Chaol estimator and Shannon estimator) were calculated using mothur (v 1.31.2). Analyses of unique OTUs and OTUs shared between the four species were conducted based on an OTU table generated by QIIME (v1.9.0). Beta diversity for both weighted and unweighted UniFrac was calculated by QIIME software (v1.9.0). Cluster analysis was preceded by principal component analysis (PCA), which was applied to reduce the dimension of the original variables using the FactoMineR package and ggplot2 
package in $\mathrm{R}$ software (v 2.15.3). Principal coordinate analysis (PCoA) was performed to obtain principal coordinates and visualize complex, multidimensional data. PCoA results were displayed by the WGCNA package, stat packages and ggplot2 package in $\mathrm{R}$ software ( $\mathrm{v}$ 2.15.3). Unweighted pair-group method with arithmetic means (UPGMA) clustering was performed as a hierarchical clustering method to interpret the distance matrix using average linkages and was conducted by QIIME software (v1.8.0). The significance of microbiota structure differentiation among groups was assessed by permutational multivariate analysis of variance (PERMANOVA) and analysis of similarities (ANOSIM) using the $\mathrm{R}$ package vegan (v2.5-6) [62]. Tax4Fun [63, 64] software was used to conduct microbial function prediction based on the nearest-neighbor method and the minimum $16 \mathrm{~S}$ rRNA sequence similarity to obtain functional annotation information.

\section{Abbreviations}

ALV-J: Avian leukosis virus J; ALV-K: Avian leukosis virus K; ANOSIM: Analysis of similarities; FMT: Fecal microbiota transplantation; GALT: Gut-associated lymphoid tissue; GIT: Gastrointestinal tract; IBDV: Infectious bursal disease virus; MDV: Marek's disease virus; MD: Marek's disease; REV: Avian reticuloendotheliosis virus; OTU: Operational taxonomic unit; PCoA: Principal coordinate analysis; PCA: Principal component analysis;

PERMANOVA: Permutational multivariate analysis of variance

\section{Acknowledgments}

Not applicable.

\section{Authors' contributions}

All authors participated in study design and planning. XW, LX, YW and XS conceived and designed the study; XW, LX acquired and extracted the data; $X W, L X$ and $Y W$ wrote the main manuscript; XS, ZW, HL1 and RH helped to draft the manuscript; XW, XL, FY and ZL contributed to the bioinformatics analysis of the sequencing data; $H L 2, Y T, X L$, and XK interpreted the data, and revised the manuscript. All authors read and approved the final manuscript.

\section{Funding}

This study was supported by the Department of Education of Henan Province (No. 18A350012), the Department of Science and Technology of Henan Province (No. 182102310098), and the Fang's family (Hong Kong) foundation. The funding agencies did not participate in study design, data collection, analysis, decision to publish or writing of the manuscript.

\section{Availability of data and materials}

The datasets used and analyzed during the current study are available from the corresponding author upon reasonable request.

\section{Ethics approval and consent to participate}

This study was ethically approved by the Henan Agricultural University's Animal Welfare and Ethical Review Board. All procedures were performed in accordance with the guidelines for the care and use of animals of Henan Agricultural University (China) and with current Chinese legislation. The farm owner gave verbal consent for the sample collections and can use chickens. As the farm is our collaborate farm, the ethics committee approved this procedure.

\section{Consent for publication}

Not applicable.

\section{Competing interests}

The authors declare that they have no competing interests.

\section{Author details}

${ }^{1}$ College of Animal Science and Veterinary Medicine, Henan Agricultural University, Zhengzhou 450000, China. ${ }^{2}$ College of Environmental and Resource Sciences, Henan Agricultural University, Zhengzhou 450000, China. ${ }^{3}$ Henan Research Center of Germplasm Resources for Poultry, Zhengzhou 450002, China. "Key Laboratory of "Runliang" Antiviral Medicines Research and Development, Institute of Drug Discovery \& Development, Zhengzhou University, Zhengzhou 450001, China.

Received: 9 November 2019 Accepted: 15 June 2020

Published online: 29 June 2020

\section{References}

1. Meng FF, Li QC, Zhang YB, Cui ZZ, Chang S, Zhao P. Isolation and characterization of subgroup J Avian Leukosis virus associated with hemangioma in commercial Hy-Line chickens. Poult Sci. 2018;97(8):2667-74.

2. Wen Y, Huang Q, Yang C, Pan L, Wang G, Qi K, Liu H. Characterizing the histopathology of natural co-infection with Marek's disease virus and subgroup J avian leucosis virus in egg-laying hens. Avian Pathol. 2018;47(1): 83-9.

3. Yang Y, Zhao J, Ma Z, Xu M, Xue J, Zhang G. Serological survey of Reticuloendotheliosis virus infection in chickens in China in 2005 to 2015. Poult Sci. 2017;96(11):3893-5.

4. Payne LN, Venugopal K. Neoplastic diseases: Marek's disease, avian leukosis and reticuloendotheliosis. Rev Sci Tec (International Office of Epizootics). 2000;19(2):544-64.

5. Payne LN, Brown SR, Bumstead N, Howes K, Frazier JA, Thouless ME. A novel subgroup of exogenous avian leukosis virus in chickens. J Gen Virol. 1991; 72(Pt 4):801-7.

6. Xu BR, Dong $W X$, Yu CM, He ZQ, Lv YL, Sun YH, Feng XY, Li N, Lee LF, Li M. Occurrence of avian leukosis virus subgroup $J$ in commercial layer flocks in China. Avian Pathol. 2004;33(1):13-7.

7. Gao YL, Qin LT, Pan W, Wang YQ, Qi XL, Gao HL, Wang XM. Avian Leukosis virus subgroup J in layer chickens, China. Emerg Infect Dis. 2010;16(10): 1637-8.

8. Gao YL, Yun BL, Qin LT, Pan W, Qu Y, Liu ZS, Wang YQ, Qi XL, Gao HL, Wang XM. Molecular epidemiology of avian leukosis virus subgroup J in layer flocks in China (vol 50, pg 953, 2012). J Clin Microbiol. 2012;50(6):2183.

9. Wang YX, Li JL, Li Y, Fang LC, Sun XL, Chang S, Zhao P, Cui ZZ. Identification of avian leukosis virus subgroup J-associated acutely transforming viruses carrying the v-src oncogene in layer chickens. J Gen Virol. 2016;97:1240-8.

10. Li HJ, Wang PK, Lin LL, Shi MY, Gu ZM, Huang T, Mo MI, Wei TC, Zhang HM, Wei P. The emergence of the infection of subgroup J avian leucosis virus escalated the tumour incidence in commercial yellow chickens in southern China in recent years. Transbound Emerg Dis. 2019;66(1):312-6.

11. Sun H, Qin M, Xiao Y, Yang F, Ni W, Liu S. Haemangiomas, leiomyosarcoma and myeloma caused by subgroup J avian leukosis virus in a commercial layer flock. Acta Vet Hung. 2010;58(4):441-51.

12. Smith $L P$, Petheridge $L$, Nair V, Wood A, Welchman D. Avian leukosis virus subgroup J-associated myelocytoma in a hobby chicken. Vet Record. 2018; 182(1):23.

13. Qu Y, Sun H, Sun M, Li D, Qin L, Gao Y, Wang X, Liu S. Sequence analysis for the complete proviral genome of avian leukosis virus subgroup J associated with haemangiomas, leiomyosarcomas and myelomas in layer flocks. Acta Vet Hung. 2012;60(3):343-54.

14. Hertig C, Coupar BE, Gould AR, Boyle DB. Field and vaccine strains of fowlpox virus carry integrated sequences from the avian retrovirus, reticuloendotheliosis virus. Virology. 1997;235(2):367-76.

15. Koo BS, Lee HR, Jeon EO, Jang HS, Han MS, Min KC, Lee SB, Kim JJ, Mo IP. An outbreak of lymphomas in a layer chicken flock previously infected with Fowlpox virus containing integrated reticuloendotheliosis virus. Avian Dis. 2013;57(4):812-7.

16. Joshi LR, Bauermann FV, Hain KS, Kutish GF, Armien AG, Lehman CP, Neiger R, Afonso CL, Tripathy DN, Diel DG. Detection of Fowlpox virus carrying distinct genome segments of Reticuloendotheliosis virus. Virus Res. 2019; 260:53-9.

17. Wozniakowski G, Mamczur A, Samorek-Salamonowicz E. Common occurrence of Gallid herpesvirus-2 with reticuloendotheliosis virus in chickens caused by possible contamination of vaccine stocks. J Appl Microbiol. 2015;118(4):803-8. 
18. Y-p Z, Bao K-y, G-r S, H-c L, H-y C, Gao Y-I, Wang X-m, C-j L. Characterization of a Gallid herpesvirus 2 strain with novel reticuloendotheliosis virus long terminal repeat inserts. Virus Genes. 2017;53(3):386-91.

19. Li JP, Dong X, Yang CH, Li QH, Cui ZZ, Chang S, Zhao P, Yu KZ, Yang HC. Isolation, identification, and whole genome sequencing of reticuloendotheliosis virus from a vaccine against Marek's disease. Poult Sci. 2015;94(4):643-9.

20. Witter RL, Peterson IL, Smith EJ, Johnson DC. Serologic evidence in commercial chicken and Turkey flocks of infection with reticuloendotheliosis virus. Avian Dis. 1982;26(4):753-62.

21. Sasaki T, Sasaki S, Koyama H. A survey of an antibody to reticuloendotheliosis virus in sera of chickens and other avian species in Japan. J Vet Med Sci. 1993;55(5):885-8.

22. Stewart B, Trautman C, Cox F, Spann H, Hardin J, Dittmar R, Edwards D. Survey of Reticuloendotheliosis virus in wild turkeys (Meleagris gallopavo) in Texas, USA. J Wildl Dis. 2019;55(3):689-93.

23. Cui ZZ, Sun SH, Zhang Z, Meng SS. Simultaneous endemic infections with subgroup J avian leukosis virus and reticuloendotheliosis virus in commercial and local breeds of chickens. Avian Pathol. 2009;38(6):443-8.

24. Dong $X$, Ju S, Zhao P, Li Y, Meng F, Sun P, Cui Z. Synergetic effects of subgroup $\mathrm{J}$ avian leukosis virus and reticuloendotheliosis virus co-infection on growth retardation and immunosuppression in SPF chickens. Vet Microbiol. 2014;172(3-4):425-31.

25. Dong X, Zhao P, Chang S, Ju S, Li Y, Meng F, Sun P, Cui Z. Synergistic pathogenic effects of co-infection of subgroup $J$ avian leukosis virus and reticuloendotheliosis virus in broiler chickens. Avian Pathol. 2015;44(1):43-9.

26. Sun GR, Zhang YP, Zhou LY, Lv HC, Zhang F, Li K, Gao YL, Qi XL, Cui HY, Wang $Y Q$, et al. Co-infection with Marek's disease virus and reticuloendotheliosis virus increases illness severity and reduces Marek's disease vaccine efficacy. Viruses-Basel. 2017;9(6):158.

27. Clavijo V, Florez MJV. Non-invited review the gastrointestinal microbiome and its association with the control of pathogens in broiler chicken production: a review. Poult Sci. 2018;97(3):1006-21.

28. Stanley D, Hughes RJ, Moore RJ. Microbiota of the chicken gastrointestinal tract: influence on health, productivity and disease. Appl Microbiol Biotechnol. 2014;98(10):4301-10.

29. Yitbarek A, Taha-Abdelaziz K, Hodgins DC, Read L, Nagy E, Weese JS, Caswell JL, Parkinson J, Sharif S. Gut microbiota-mediated protection against influenza virus subtype H9N2 in chickens is associated with modulation of the innate responses. Sci Rep. 2018;8:12.

30. Roxas JL, Viswanathan VK. Modulation of intestinal paracellular transport by bacterial pathogens. Compr Physiol. 2018;8(2):823-42.

31. Wong ACN, Vanhove AS, Watnick PI. The interplay between intestinal bacteria and host metabolism in health and disease: lessons from Drosophila melanogaster. Dis Model Mech. 2016;9(3):271-81.

32. Sun $\mathrm{XL}$, Jia ZQ. Microbiome modulates intestinal homeostasis against inflammatory diseases. Vet Immunol Immunopathol. 2018;205:97-105.

33. Cisek AA, Binek M. Chicken intestinal microbiota function with a special emphasis on the role of probiotic bacteria. Pol J Vet Sci. 2014;17(2):385-94.

34. Stanley D, Denman SE, Hughes RJ, Geier MS, Crowley TM, Chen HL, Haring VR, Moore RJ. Intestinal microbiota associated with differential feed conversion efficiency in chickens. Appl Microbiol Biotechnol. 2012;96(5):1361-9.

35. Ding JM, Dai RH, Yang LY, He C, Xu K, Liu SY, Zhao WJ, Xiao L, Luo LX, Zhang $Y$, et al. Inheritance and establishment of gut microbiota in chickens. Front Microbiol. 2017:8:11.

36. Wang $Y$, Xu L, Sun X, Wan X, Sun G, Jiang R, Li W, Tian Y, Liu X, Kang X. Characteristics of the fecal microbiota of high- and low-yield hens and effects of fecal microbiota transplantation on egg production performance. Res Vet Sci. 2020;129:164-73.

37. Day JM, Oakley BB, Seal BS, Zsak L. Comparative analysis of the intestinal bacterial and RNA viral communities from sentinel birds placed on selected broiler chicken farms. PLoS One. 2015;10(1):15.

38. Ma X, Wang Q, Li H, Xu C, Cui N, Zhao X. 16 S rRNA genes Illumina sequencing revealed differential cecal microbiome in specific pathogen free chickens infected with different subgroup of avian leukosis viruses. Vet Microbiol. 2017;207:195-204.

39. Day JM, Zsak L. Recent Progress in the characterization of avian enteric viruses. Avian Dis. 2013;57(3):573-80.

40. Liu LY, Lin LL, Zheng LN, Tang H, Fan XZ, Xue NG, Li M, Liu M, Li XY. Cecal microbiome profile altered by salmonella enterica, serovar Enteritidis inoculation in chicken. Gut Pathogens. 2018;10:14.
41. Macdonald SE, Nolan MJ, Harman K, Boulton K, Hume DA, Tomley FM, Stabler RA, Blake DP. Effects of Eimeria tenella infection on chicken caecal microbiome diversity, exploring variation associated with severity of pathology. PLoS One. 2017;12(9):17.

42. Li L, Kubasova T, Rychlik I, Hoerr FJ, Rautenschlein S. Infectious bursal disease virus infection leads to changes in the gut associated-lymphoid tissue and the microbiota composition. PLoS One. 2018;13(2):e0192066.

43. Perumbakkam S, Hunt HD, Cheng HH. Marek's disease virus influences the core gut microbiome of the chicken during the early and late phases of viral replication. FEMS Microbiol Ecol. 2014;90(1):300-12.

44. Liu H, Ma K, Liu M, Yang C, Huang X, Zhao Y, Qi K. Histologic findings and viral antigen distribution in natural coinfection of layer hens with subgroup J avian leukosis virus, Marek's disease virus, and reticuloendotheliosis virus. J Vet Diagn Investig. 2019;31(5):761-5.

45. Zhou J, Zhao GL, Wang XM, Du XS, Su S, Li CG, Nair V, Yao YX, Cheng ZQ. Synergistic viral replication of Marek's Disease virus and avian leukosis virus subgroup $\mathrm{J}$ is responsible for the enhanced pathogenicity in the superinfection of chickens. Viruses. 2018;10(5):271.

46. Wang Y, Fang L, Li J, Li Y, Cui S, Sun X, Chang S, Zhao P, Cui Z. Rescue of avian leukosis subgroup-J-associated acutely transforming viruses carrying different lengths of the $\mathrm{v}$-fps oncogene and analysis of their tumorigenicity. Arch Virol. 2016;161(12):3473-81.

47. Li HX, Liu XL, Chen FY, Zuo KJ, Wu C, Yan YM, Chen WG, Lin WC, Xie QM. Avian influenza virus subtype H9N2 affects intestinal microbiota, barrier structure injury, and inflammatory intestinal disease in the chicken ileum. Viruses-Basel. 2018;10(5):14.

48. Hopkins EGD, Roumeliotis TI, Mullineaux-Sanders C, Choudhary JS, Frankel G. Intestinal Epithelial Cells and the Microbiome Undergo Swift Reprogramming at the Inception of Colonic Citrobacter rodentium Infection. mBio. 2019;10(2):e00062-19.

49. Wang G, Wang Y, Yu L, Jiang Y, Liu J, ZJMP C. New pathogenetic characters of reticuloendotheliosis virus isolated from Chinese partridge in specificpathogen-free chickens. Microb Pathog. 2012;53(2):57-63.

50. Lin L, Wang P, Yang Y, Li H, Huang T, Wei P. Full-length genome sequence analysis of four subgroup $J$ avian leukosis virus strains isolated from chickens with clinical hemangioma. Virus Genes. 2017;53(6):868-75.

51. Guo HJ, Hong-Mei LI, Cheng ZQ, Liu JZ, ZZJASiC C. Influence of REV and ALV-J Co-Infection on Immunologic Function of T Lymphocytes and Histopathology in Broiler Chickens. Agricultural Sciences in China. 2010; 9(11):0-1676.

52. Mohd Shaufi M, Sieo C, Chong C, Gan H, Ho YJGP. Deciphering chicken gut microbial dynamics based on high-throughput $16 \mathrm{~S}$ rRNA metagenomics analyses. Gut Pathog. 2015;7(1):1-12.

53. Cui N, Wang XZ, Wang Q, Li HM, Wang FK, Zhao XM. Effect of dual infection with Eimeria tenella and subgroup J avian Leukosis virus on the Cecal microbiome in specific-pathogen-free chicks. Front Vet Sci. 2017;4:10.

54. Smith LM, Brown SR, Howes K, et al. Development and application of polymerase chain reaction (PCR) tests for the detection of subgroup $J$ avian leukosis virus. Virus Res. 1998;54(1):87-98.

55. Aly MM, Smith EJ, Fadly AM. Detection of reticuloendotheliosis virus infection using the polymerase chain reaction. Avian Pathol. 1993;22(3):543-54.

56. Davidson I, Borovskaya A, Perl S, Malkinson M. Use of the polymerase chain reaction for the diagnosis of natural infection of chickens and turkeys with Marek's disease virus and reticuloendotheliosis virus. Avian Pathol. 1995;24(1):69-94.

57. Caporaso JG, Kuczynski J, Stombaugh J, Bittinger K, Bushman FD, Costello EK, Fierer N, Peña AG, Goodrich JK, Gordon Jl, et al. QIIME allows analysis of highthroughput community sequencing data. Nat Methods. 2010;7(5):335-6.

58. Gill SR, Pop M, Deboy RT, Eckburg PB, Turnbaugh PJ, Samuel BS, Gordon II, Relman DA, Fraser-Liggett CM, Nelson KE. Metagenomic analysis of the human distal gut microbiome. Science (New York, NY). 2006;312(5778):1355-9.

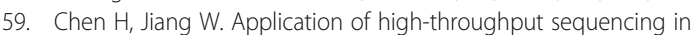
understanding human oral microbiome related with health and disease. Front Microbiol. 2014;5:508.

60. Magoč T, Salzberg SL. FLASH: fast length adjustment of short reads to improve genome assemblies. Bioinformatics (Oxford, England). 2011;27(21): 2957-63.

61. Edgar RC. Search and clustering orders of magnitude faster than BLAST. Bioinformatics (Oxford, England). 2010;26(19):2460-1.

62. Kambura AK, Mwirichia RK, Kasili RW, Karanja EN, HIJBM B. Bacteria and Archaea diversity within the hot springs of Lake Magadi and Little Magadi in Kenya. BMC Microbiol. 2016;16(1):136. 
63. Wildenhain J, Fitzgerald N, Tyers M. MolClass: a web portal to interrogate diverse small molecule screen datasets with different computational models. Bioinformatics (Oxford, England). 2012;28(16):2200-1.

64. Aßhauer KP, Wemheuer B, Daniel R, Meinicke P. Tax4Fun: predicting

functional profiles from metagenomic 165 rRNA data. Bioinformatics (Oxford, England). 2015;31(17):2882-4

\section{Publisher's Note}

Springer Nature remains neutral with regard to jurisdictional claims in published maps and institutional affiliations.

Ready to submit your research? Choose BMC and benefit from:

- fast, convenient online submission

- thorough peer review by experienced researchers in your field

- rapid publication on acceptance

- support for research data, including large and complex data types

- gold Open Access which fosters wider collaboration and increased citations

- maximum visibility for your research: over $100 \mathrm{M}$ website views per year

At $\mathrm{BMC}$, research is always in progress.

Learn more biomedcentral.com/submissions 